\title{
Wearable Inkjet-Printed Wideband Antenna by using Miniaturized AMC for Sub-GHz Applications
}

\author{
Simone Genovesi, Member, IEEE, Filippo Costa, Member, IEEE, Filippo Fanciulli, and Agostino \\ Monorchio, Fellow, IEEE
}

\begin{abstract}
A novel compact wearable antenna printed on paper is presented. The antenna comprises a non-resonant coplanar monopole antenna placed on a miniaturized AMC (total size of $0.14 \lambda_{0} \times 0.14 \lambda_{0} \times 0.01 \lambda_{0}$ at $\left.700 \mathrm{MHz}\right)$. The manufacturing process only requires a commercial desktop inkjet printer and conductive nano-ink. The effectiveness of the proposed configuration (monopole above AMC) with respect to the unloaded monopole or to the monopole above a metallic ground plane is proved with on-body measurements in the sub-GHz band.
\end{abstract}

Index Terms-Inkjet antenna, small antenna, Artificial Magnetic Conductor (AMC), wearable antenna, on body, subGHz, wireless applications, miniaturization, wideband.

\section{INTRODUCTION}

$\mathrm{W}$ earable devices are receiving more and more attention by the ICT consumer market for on-body communication as well as for connecting to wireless networks; therefore, their rapid and pervasive spreading is expected in the next years [1]. The IEEE 802.15 has recently regulated the various paradigms of on body, off body and in body communications. Within this framework, several technological solutions have been proposed in order to fabricate antennas that can fit on a human body. Since the use of rigid dielectric substrates commonly employed in standard PCB lithographic processes does not provide the necessary degrees of freedom in terms of integration and wearability, organic and polymer substrates [2] have been considered as promising candidates for realizing light and flexible radiators. For example, textile antennas have been proposed in [3], [4] whereas antennas printed on paper have been presented in [6], [7]. These antennas are widely employed for telecommunications [8] but other applications involve telemedicine [9], radio frequency identification (RFID) [10],[11], sensors [12], and energy harvesting [13]. One of the most challenging aspects of designing wearable antennas is avoiding as much as possible the negative effect of the interaction between the antenna and the dissipative biological tissue. In fact, the human body detunes the antenna and absorbs a large amount of the radiated power, thus reducing the gain. It is therefore necessary to isolate the antenna in order to preserve its radiation characteristic as much as possible and to prevent hazardous biological effects. Artificial Magnetic Conductors (AMCs) have been successfully employed to cope with this problem for their capability of isolating an antenna from the surrounding environment [9], [7], [14].
It is interesting to notice that few of the proposed wearable antennas work at frequencies lower than $1 \mathrm{GHz}$ where some frequency ranges are allocated as in the case of some LTE channels (700-960 MHz), ISM and GSM bands (900 MHz), wireless sensor networks [3], [10], [13]-[15]. This is probably due to the cumbersome dimensions of an antenna working in the sub-GHz region. However, wider range, better in-building penetration and lower operative cost with respect to higher frequencies render them a promising asset in the wireless arena [16].

In this letter, a wearable radiating structure realized on a paper substrate by using conductive nanoink is presented. A non-resonant coplanar monopole antenna has been closely placed on a miniaturized AMC suitably designed to limit the overall footprint of the radiating structure to a limited area of $0.14 \lambda_{0} \times 0.14 \lambda_{0}$. This compact radiating structure is able to operate in a sub-GHz band and it can be manufactured by using a commercial desktop inkjet printer. This letter is organized as follows. Section II describes the miniaturization concept applied to the AMC design. Section III illustrates the proposed antenna configuration. On body measurements of the antenna are presented in Section IV whereas conclusions are drawn in Section V.

\section{MINIATURIZED AMC CONCEPT}

An Artificial Magnetic Conductor (AMC) is a periodic planar array of conductive elements printed on grounded dielectric substrates. By employing an AMC, it is possible to place a planar electric source close to metasurface without compromising its radiating properties. Although the shape of the periodic elements can vary from dipole to patches or more complex geometries [17] the physical size of the unit cell is in the order of half a wavelength. Since it is convenient to minimize the size of the periodic unit cell constituting the AMC to better approximate a homogenous medium, the miniaturization of the periodic element is a key issue. This is particularly important for antennas operating at sub $\mathrm{GHz}$ regime where even half a wavelength can be an unpractical size in some applications.

Convoluted elements and space-filling curves have been employed to reduce the size of the AMC unit cells [17]-[20] but these solutions affect the operating bandwidth. A most efficient solution in terms of bandwidth and space reduction has been achieved by a $90^{\circ}$-rotational fourth-fold symmetric square unit cell where the periodic element extends beyond the periodicity [20]-[24]. The unit cell considered in this work comprises a convoluted eight-arm element whose overall 
dimension $H=5.5 \mathrm{~cm}$ exceeds the periodicity $D$ of the AMC (Fig. 1). As demonstrated in [25], a non-resonant antenna closely placed on an AMC can be matched within a band comprising the AMC resonance frequency thus providing a compact radiating element. For this reason, a miniaturized AMC screen has been designed to resonate at $700 \mathrm{MHz}$ and manufactured by using silver nanoink printed on a paper substrate to provide the necessary tuning of the non-resonant antenna placed on it. The choice of organic substrates is favorable since it allows cheaper, lighter and flexible designs than common silicon or dielectric substrates such as FR4.
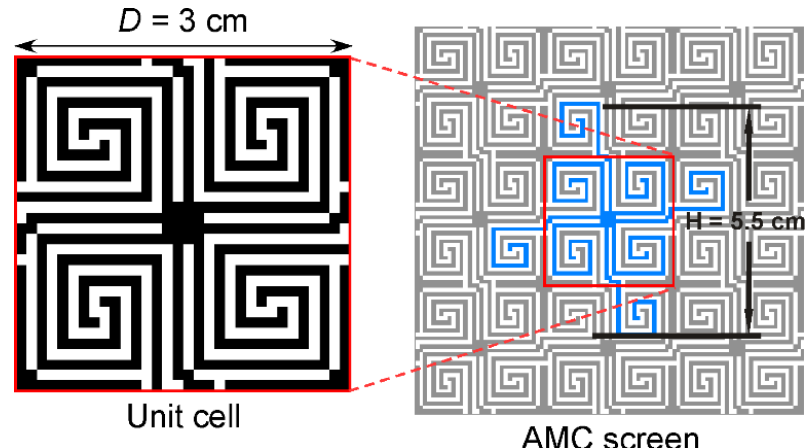

Fig. 1. Top view of the AMC screen. The zoomed area remarks the periodic square unit cell with periodicity $D=3 \mathrm{~cm}$. The footprint of the eight-arm element is $H=5.5 \mathrm{~cm}$ (highlighted in blue).

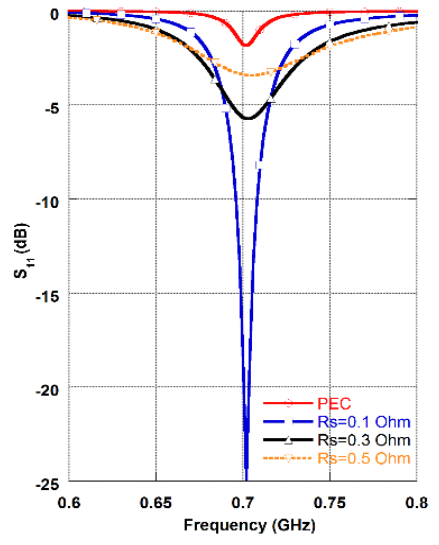

(a)

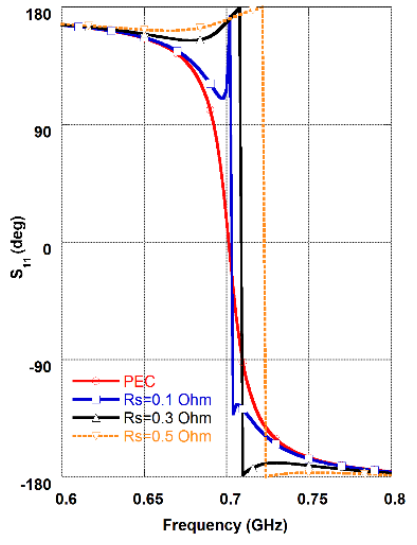

(b)
Fig. 2. Frequency response of the AMC screen: $S_{11}$ amplitude (a) and phase (b).

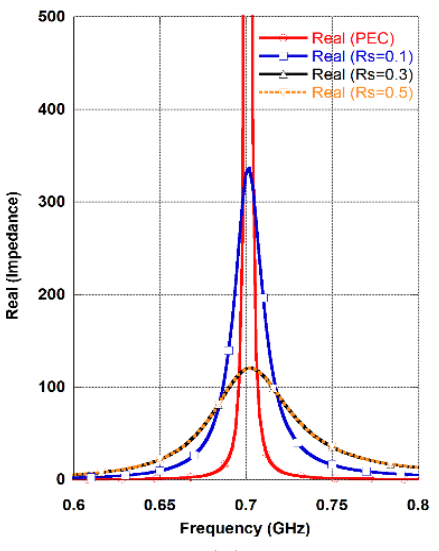

(a)

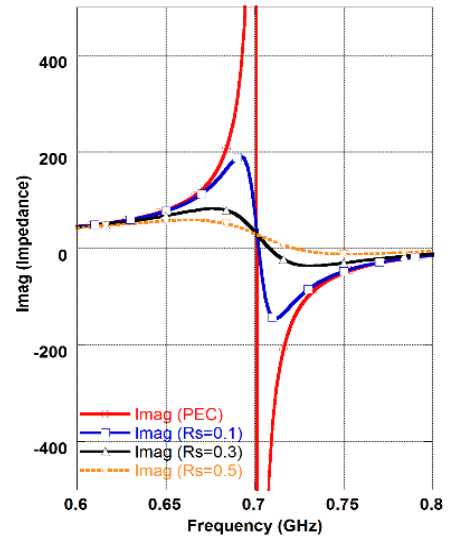

(b)
Fig. 3. Frequency response of the AMC screen: real part (a) and imaginary part $(b)$ of the equivalent AMC impedance.
They can provide flexibility and easier integration in garments or wearability. Moreover, inkjet printing avoids any lithographic process thus speeding up the prototyping phase. In addition, it also drastically reduce the production cost since no mask has to be prepared and no chemical etching is required. The proposed AMC stack up comprises a first sheet of resin paper where the periodic surface is printed, a $5 \mathrm{~mm}$ thick polycarbonate substrate $\left(\varepsilon_{\mathrm{r}}=2.56, \tan \delta=0.01\right)$ and another paper sheet where a uniform deposition of silver ink acts as a ground plane. The final AMC design has a unit cell with periodicity $D=3 \mathrm{~cm}\left(0.07 \lambda_{0}\right)$ and a total thickness of $0.012 \lambda_{0}$. The frequency response of the periodic surface has been analyzed by using a numerical code implementing a Periodic Method of Moments [26] that is able to analyze PEC surfaces as well as lossy ones. The reflection coefficient $S_{11}$ is reported in Fig. 2 in both amplitude and phase for different value of ink resistance. It is apparent that moderate losses are introduced by the silver nano ink around the resonance frequency with respect to a AMC screen realized with metal (PEC case) whereas the steepness of the reflection coefficient phase is increasing with losses [27]. It is also interesting to look at the equivalent impedance exhibited by the printed AMC screen (Fig. 3). The level of matching of the nonresonant antenna benefits from the finite values of the real and imaginary part of the AMC impedance [25].

\section{PRINTED ANTENNA DESIGN AND FABRICATION}

\section{A. Material characterization}

The permittivity of the employed $135 \mu$ m-thick resin-coated paper (Mitsubishi NB-RC-3GR120) has been evaluated by applying the Nicholson Technique to transmission reflection measurements obtained with the WR187 $(4.4 \mathrm{GHz}-5.0 \mathrm{GHz})$ and the WR137 (5.0 GHz-7.5 GHz) waveguides. The value of permittivity was found equal to 3.5 . This value is consistent with the data in open literature [7] and it can be reasonably used even in the sub-GHz regime since no relaxation effect is observed.

Next, the resistivity of the adopted conductive silver nanoink (Mitsubishi NBSIJ-MU01) has been estimated as well by using a transmission reflection technique performed in wa waveguide setup [28]. More in detail, a commercial desktop printer with a piezoelectric print head was used to fabricate the sample under test consisting in a uniform deposition of ink on the paper. No sintering process has been used since the ink cures at room temperature. The print quality did not significantly affect the estimated sheet resistance since the inferred value remains almost constant by using the fast print, normal and high quality. The retrieved ink resistance $R_{s}$ was determined equal to $0.3 \mathrm{Ohm} / \mathrm{sq}$ for a trace thickness of $1 \mathrm{um}$. Then, several tests on the achievable print resolution have been performed by drawing two parallel lines with distances decreasing from $500 \mu \mathrm{m}$ up to $50 \mu \mathrm{m}$. Microscope enlargements showed a greater definition on the horizontal lines than on vertical ones, as expected from nominal values of our printer (Brother DCP-J152W) and the minimum gap acceptable to calculate the available resolution was determined equal to $150 \mu \mathrm{m}$. 


\section{B. Geometry and Stack up}

The employed antenna is the non-resonant monopole reported in Fig. 4. The antenna is realized with the nano ink printed on a resin-coated paper and the most relevant parameters are $P=55 \mathrm{~mm}, A=10 \mathrm{~mm}, B=26.5 \mathrm{~mm}$, $W_{l}=42 \mathrm{~mm}$ and $W_{2}=29 \mathrm{~mm}$. At the natural resonance $(3.5 \mathrm{GHz})$ the printed antenna gain is $3.7 \mathrm{dBi}$ and the radiated field polarized along $y$-axis (Xpol.<-30 dB). Conventional soldering techniques are not suitable for connecting components to inkjet circuits because the ink and the paper substrate cannot tolerate soldering temperatures. For this application both a two-part thermal conductive epoxy loaded with silver particles (MG Chemicals) and an electrically adhesive conductive tape (3M 9712) were tested for fixing the SMA connector. Both of them may introduce relevant additional losses since they do not guarantee an ideal electric connection.

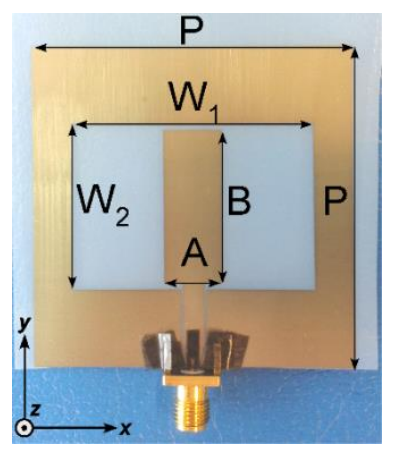

(a)

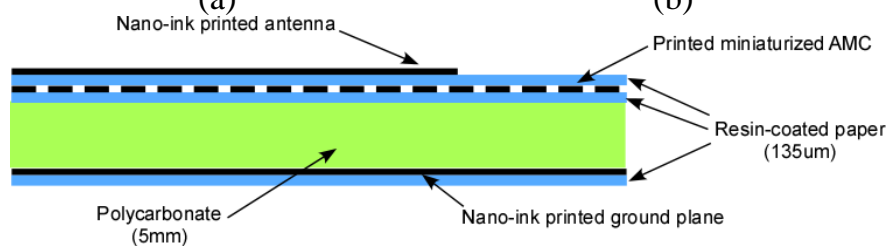

(c)
Fig. 4. View of the manufactured antenna prototype. (a) Monopole antenna and (b) a top view of the fabricated AMC screen $(L=6 \mathrm{~cm})$ place below the monopole antenna. (c) Stack-up of the overall structure.

The finite AMC screen placed underneath the antenna is composed by an array of $2 \times 2$ unit cells (Fig. $4 b$ ), hence the composed radiator has a very compact size of $6 \mathrm{~cm} \times 6 \mathrm{~cm}$ $\left(0.14 \lambda_{0} \times 0.14 \lambda_{0}\right)$. It is important to underline that only the silver nanoink has been used to print the antenna, the AMC as well as the ground plane since no copper surface was employed as illustrated in Fig. 4c.

\section{Effect of Ink Resistivity and SMA Connection}

The impact of a different value of the ink resistance $R_{s}$ has been investigated in Fig. 5 by using CST Microwave Studio. It is expected that the antenna matching is determined by the coupling with the resistive AMC [25]. It is apparent that only the deepness of the resonant peak changes whereas the resonance frequency does not significantly vary. The comparison among the simulations and the measurement shows that the $-10 \mathrm{~dB}$ bandwidth estimated by numerical simulations is narrower than measured one and the resonant peak is shifted. The same shift has been found both in the case of the conductive epoxy and conductive tape were used for mounting the SMA connector and it is probably due to parasitic effects and imperfect contact that was no possible to consider in the simulations. SMA connection is surely one of the weak points of these type of antennas and research is currently being focused on this aspect.

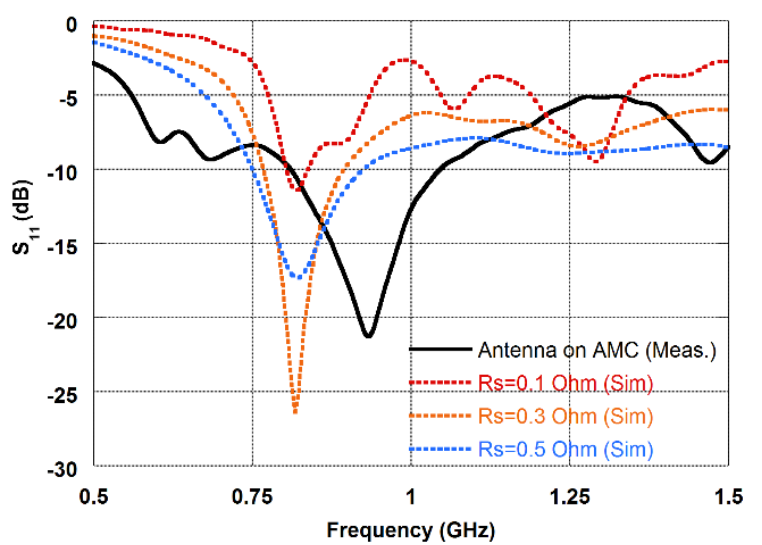

Fig. 5. Measured reflection coefficient of the antenna on AMC compared to simulations for different values of ink resistance.

\section{ON BODY EXPERIMENTAL RESULTS}

When an antenna is accommodated close to a human body, a degradation of the performance in terms of gain and efficiency is well known. The harmful effects of the lossy human body are usually attenuated by using antennas loaded with a metallic ground. In order to assess the advantage of using the AMC ground plane instead of using a PEC ground, some experimental tests have been performed by accommodating different antenna configurations on the torso of a human body. An estimation of the realized gain has been carried out by performing comparative measurements of the power received with a log-periodic antenna placed at a distance of Dist $=2.0 \mathrm{~m}$ from the antennas under test $\left(\mathrm{S}_{21}\right)$. Reflection coefficient of the on body antenna $\left(S_{11}\right)$ has been also measured. In Fig. 6 the results for the monopole placed on the AMC ground plane and on the PEC ground plane are compared. In the former, the coplanar monopole has been placed on the miniaturized AMC screen as reported in Errore. L'origine riferimento non è stata trovata. and applied to the body. In the latter, the stack up remains unchanged (the paper sheet on which the periodic surface is printed is replaced by a non-printed paper sheet). This comparison is considered fair since it provides the same overall thickness of the radiating structure. Looking at the $S_{11}$, it is evident that the antenna on the PEC ground is not matched in the sub $\mathrm{GHz}$ region whereas the antenna on the AMC exhibits a $-10 \mathrm{~dB}$ bandwidth spanning from almost $750 \mathrm{MHz}$ up to more than $1 \mathrm{GHz}$. More interestingly, in the same aforementioned bandwidth, the level of $S_{21}$ provided by the antenna on the AMC is higher than the one offered by the antenna on the PEC ground. More interestingly, in the same aforementioned bandwidth, the level of $S_{21}$ provided by the antenna on the AMC is higher than the one offered by the antenna on the PEC ground. The improvement in the $S_{21}$ of the antenna on the miniaturized AMC with respect to the antenna on PEC (AMCvsPEC) and with respect to the unloaded monopole (AMCvsMonopole) for 
the on body case is reported in Fig. 7. It is highlighted that for both cases the improvement is obtained in a large sub-GHz bandwidth. This proves the effectiveness of the proposed structure in providing a better link and lower coupling with the user.

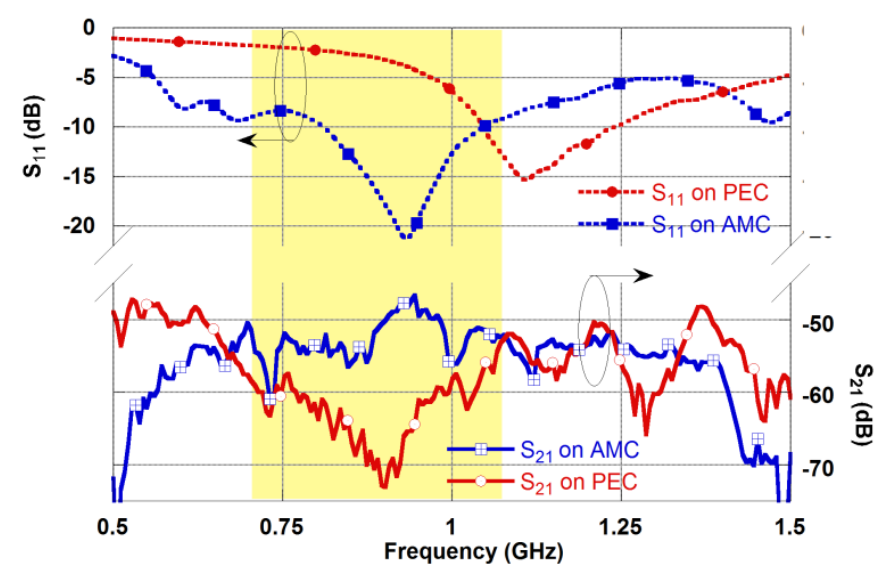

Fig. 6. Comparison between on body measurements of the coplanar monopole antenna placed on AMC or on a PEC ground plane.

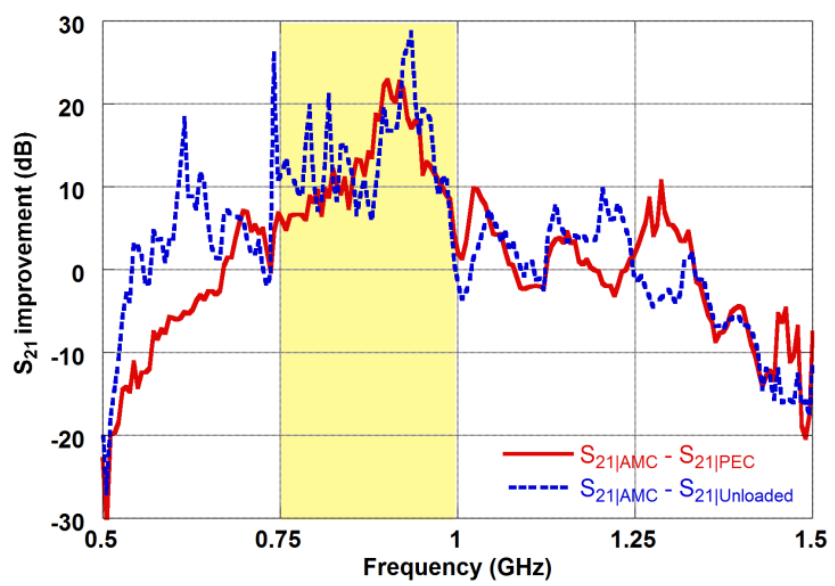

Fig. 7. $S_{21}$ improvement of the antenna on the miniaturized AMC with respect to the antenna on PEC (AMCvsPEC) and with respect to the monopole alone (AMCvsMonopole) for the on body case.

\section{CONCLUSION}

A compact radiating structure realized with inkjet printing technology has been presented. The proposed antenna is realized on flexible resin-coated paper and is particularly suitable for wearable application due to its small size. The footprint has been minimized by resorting to a miniaturized AMC screen. The proposed structure limits the interaction of the antenna with the user as an antenna on a ground plane does, but it is able to provide a better radiation performance. Measurements on manufactured prototypes assess the benefit of employing the miniaturized AMC screen in antennas for on body communications.

\section{REFERENCES}

[1] C. C. Y. Poon, B. P. L. Lo, M. R. Yuce, A. Alomainy, and Y. Hao, "Body Sensor Networks: In the Era of Big Data and Beyond," Biomed. Eng. IEEE Rev. In, vol. 8, pp. 4-16, 2015.

[2] M. Stoppa and A. Chiolerio, "Wearable Electronics and Smart Textiles: A Critical Review," Sensors, vol. 14, no. 7, pp. 11957-11992, Jul. 2014.
[3] M. Hirvonen, C. Bohme, D. Severac, and M. Maman, "On-Body Propagation Performance With Textile Antennas at $867 \mathrm{MHz}$," IEEE Trans. Antennas Propag., vol. 61, no. 4, pp. 2195-2199, Apr. 2013.

[4] W. G. Whittow, A. Chauraya, J. C. Vardaxoglou, Y. Li, R. Torah, K. Yang, S. Beeby, and J. Tudor, "Inkjet-Printed Microstrip Patch Antennas Realized on Textile for Wearable Applications," IEEE Antennas Wirel. Propag. Lett., vol. 13, pp. 71-74, 2014.

[5] L. Vallozzi, P. Van Torre, C. Hertleer, H. Rogier, M. Moeneclaey, and J. Verhaevert, "Wireless Communication for Firefighters Using DualPolarized Textile Antennas Integrated in Their Garment," IEEE Trans. Antennas Propag., vol. 58, no. 4, pp. 1357-1368, Apr. 2010.

[6] B. S. Cook and A. Shamim, "Inkjet Printing of Novel Wideband and High Gain Antennas on Low-Cost Paper Substrate," IEEE Trans. Antennas Propag., vol. 60, no. 9, pp. 4148-4156, Sep. 2012.

[7] S. Kim, Y.-J. Ren, H. Lee, A. Rida, S. Nikolaou, and M. M. Tentzeris, "Monopole Antenna With Inkjet-Printed EBG Array on Paper Substrate for Wearable Applications," IEEE Antennas Wirel. Propag. Lett., vol. 11, pp. 663-666, 2012.

[8] E. K. Kaivanto, M. Berg, E. Salonen, and P. de Maagt, "Wearable Circularly Polarized Antenna for Personal Satellite Communication and Navigation," IEEE Trans. Antennas Propag., vol. 59, no. 12, pp. 44904496, Dicembre 2011.

[9] H. R. Raad, A. I. Abbosh, H. M. Al-Rizzo, and D. G. Rucker, "Flexible and Compact AMC Based Antenna for Telemedicine Applications," IEEE Trans. Antennas Propag., vol. 61, no. 2, pp. 524-531, Feb. 2013.

[10] S. Manzari, C. Occhiuzzi, and G. Marrocco, "Feasibility of BodyCentric Systems Using Passive Textile RFID Tags," IEEE Antennas Propag. Mag., vol. 54, no. 4, pp. 49-62, Agosto 2012.

[11] A. Rida, L. Yang, R. Vyas, and M. M. Tentzeris, "Conductive InkjetPrinted Antennas on Flexible Low-Cost Paper-Based Substrates for RFID and WSN Applications," IEEE Antennas Propag. Mag., vol. 51, no. 3, pp. 13-23, Jun. 2009.

[12] L. Zhang, Z. Wang, and J. L. Volakis, "Textile Antennas and Sensors for Body-Worn Applications," IEEE Antennas Wirel. Propag. Lett., vol. 11, pp. 1690-1693, 2012.

[13] G. Monti, L. Corchia, and L. Tarricone, "UHF Wearable Rectenna on Textile Materials," IEEE Trans. Antennas Propag., vol. 61, no. 7, pp. 3869-3873, Jul. 2013.

[14] S. Zhu and R. Langley, "Dual-Band Wearable Textile Antenna on an EBG Substrate," IEEE Trans. Antennas Propag., vol. 57, no. 4, pp. 926-935, Apr. 2009.

[15] M. R. Islam and M. Ali, "A 900 MHz Beam Steering Parasitic Antenna Array for Wearable Wireless Applications," IEEE Trans. Antennas Propag., vol. 61, no. 9, pp. 4520-4527, Sep. 2013.

[16] D. Meyer, "The desirability of sub-1 GHz spectrum," http://www.rcrwireless.com/20131106/policy/analyst-angle-thedesirability-of-sub-1-ghz-spectrum.

[17] S. Genovesi, A. Monorchio, R. Mittra, and G. Manara, "A Subboundary Approach for Enhanced Particle Swarm Optimization and Its Application to the Design of Artificial Magnetic Conductors," IEEE Trans. Antennas Propag., vol. 55, no. 3, pp. 766-770, Mar. 2007.

[18] F. Huang, J. C. Batchelor, and E. A. Parker, "Interwoven convoluted element frequency selective surfaces with wide bandwidths," Electron. Lett., vol. 42, no. 14, pp. 788-790, Jul. 2006.

[19] B. Sanz-Izquierdo, E. A. Parker, J.-B. Robertson, and J. C. Batchelor, "Singly and Dual Polarized Convoluted Frequency Selective Structures," IEEE Trans. Antennas Propag., vol. 58, no. 3, pp. 690-696, Mar. 2010.

[20] J. McVay, N. Engheta, and A. Hoorfar, "High impedance metamaterial surfaces using Hilbert-curve inclusions," IEEE Microw. Wirel. Compon. Lett., vol. 14, no. 3, pp. 130-132, Mar. 2004.

[21] S. Barbagallo, A. Monorchio, and G. Manara, "Small periodicity FSS screens with enhanced bandwidth performance," Electron. Lett., vol. 42, no. 7, pp. 382-384, 2006

[22] A. Vallecchi and A. G. Schuchinsky, "Entwined Planar Spirals for Artificial Surfaces," IEEE Antennas Wirel. Propag. Lett., vol. 9, pp. 994-997, 2010.

[23] E. Carrubba, S. Genovesi, A. Monorchio, and G. Manara, "AMC-based low profile antennas for 4G communication services," in 2007 IEEE Antennas and Propagation Society International Symposium, 2007, pp. 3364-3367.

[24] Z. Bayraktar, J. P. Turpin, and D. H. Werner, "Nature-Inspired Optimization of High-Impedance Metasurfaces With Ultrasmall Interwoven Unit Cells," IEEE Antennas Wirel. Propag. Lett., vol. 10, pp. 1563-1566, 2011. 
[25] G. Bianconi, F. Costa, S. Genovesi, and A. Monorchio, "Optimal design of dipole antennas backed by a finite high-impedance screen," Prog. Electromagn. Res. C, vol. 18, pp. 137-151, 2011.

[26] R. Mittra, C. H. Chan, and T. Cwik, "Techniques for analyzing frequency selective surfaces-a review," Proc. IEEE, vol. 76, no. 12, pp. 1593-1615, Dec. 1988.

[27] F. Costa, S. Genovesi, A. Monorchio, and G. Manara, "A Circuit-Based Model for the Interpretation of Perfect Metamaterial Absorbers," Antennas Propag. IEEE Trans. On, vol. 61, no. 3, pp. 1201-1209, Mar. 2013.

[28] F. Costa, "Surface Impedance Measurement of Resistive Coatings at Microwave Frequencies," IEEE Trans. Instrum. Meas., vol. 62, no. 2, pp. 432-437, Feb. 2013. 